\title{
Stochastic dominance relations for generalised parametric distributions obtained through composition
}

\author{
Tommaso Lando ${ }^{1,2}$ (D) Lucio Bertoli-Barsotti ${ }^{1}$
}

Received: 15 May 2019 / Accepted: 21 July 2020 / Published online: 27 August 2020

(c) The Author(s) 2020

\begin{abstract}
Investigating stochastic dominance within flexible multi-parametric families of distributions is often complicated, owing to the high number of parameters or non-closed functional forms. To simplify the problem, we use the T-X method, making it possible to obtain generalised models through the composition of cumulative distributions and quantile functions. We derive conditions for the second-order stochastic dominance and for the increasing convex order within multi-parametric families in two steps, namely: (i) breaking them down via the T-X approach and (ii) checking dominance conditions of the (more) manageable distributions composing the model. We apply our method to some special distributions and focus on the beta-generated family, which enables the comparisons of order statistics of i.i.d. samples from (possibly) different random variables.
\end{abstract}

Keywords Stochastic dominance $\cdot \mathrm{T}-\mathrm{X}$ family $\cdot$ Generalised distributions $\cdot$ Generalised beta $\cdot$ Order statistics

Mathematics Subject Classification 60E15 · 62XX · 60E05

\section{Introduction}

Stochastic orders are primary tools in ranking probability distributions based on some preference relations [36]. Owing to several applications of ordering theory in areas such as economics, econometrics, and finance, the identification of stochastic orders within flexible generalised families of distributions (i.e. suitable to approximate a wide range of phenomena) is a relevant issue.

The literature on stochastic orders contains several studies aimed at deriving sufficient conditions for the second-order stochastic dominance (SSD) and the closely related Lorenz order (LO) for some basic parametric families. Many such conditions can be derived by what we denote as the single-crossing property. This is a fundamental result ascribable to [20] that

Tommaso Lando

tommaso.lando@unibg.it

1 University of Bergamo, Bergamo, Italy

2 VŠB-TU Ostrava, Ostrava, Czech Republic 
has also been presented elsewhere [17,33]. This property provides a sufficient condition for SSD when distributions cross once, which turns out to be especially simple to verify for basic models having few parameters. When the single-crossing condition is not directly verifiable, we rely on alternative conditions that must be verified with densities $[11,33,35]$.

Most generalised models are generally not easily tractable owing to the large number of parameters or non-closed functional forms. Thus, deriving dominance conditions may be an issue with the methods currently available. For instance, some authors derived LO conditions within (and between) some generalised size distributions with remarkable computational effort [12,21,22,34,40-43].

Regarding generalised parametric models, the so-called $T-X$ family, recently introduced by [5], provides an interesting modern method for generating distributions by composing distributions and quantile functions [2]. Such an approach makes it possible to add parameters to an existing model to obtain a new and more flexible one $[3,4,6,14,15]$. Although the method was originally introduced to compose new distributions, we use it to break down existing ones into functional compositions with certain characteristics. In fact, the T-X approach also makes it possible to decompose most existing distributions, e.g. the beta-generated (BG) family of [19], the generalised beta of the first and second kind (GB1 and GB2; [29], which are the main distributions for modelling size phenomena on bounded or unbounded support, respectively, and can generate the beta-type and gamma-type special-case distributions, including the Singh-Maddala, the Dagum or Burr (III), the Pareto (II), the standard beta, the beta of the second kind (B2), the log-logistic, the generalised gamma, the gamma and the Weibull [22]), and many others [2].

In Sect. 3, we extend a result by [26] and we provide simple theorems for deriving sufficient dominance conditions within multiparametric distributions by breaking them down via the T$\mathrm{X}$ method. Consequently, our approach checks certain conditions on the functions composing the model and avoids directly handling the composite distribution function, which is generally more difficult to deal with. We investigate the conditions for the SSD, the first-order stochastic dominance (FSD) and the increasing convex order (ICX). The method covers a wide range of applications, because it can be applied with reasonable simplicity to all distributions obtained through composition.

In Sect. 4 we derive dominance conditions for some special multi-parametric families. In particular, we focus on the BG family. Interestingly, as we show in Sect. 5, the distributions of order statistics of i.i.d. samples, from any underlying distribution $X$, belong to the BG class. This makes it possible to compare order statistics in terms of SSD, in different sampling situations. Stochastic comparisons of order statistics are particularly relevant in reliability theory, where order statistics may represent the waiting time until less than $k$ components are still functioning, in a system of $n$ components. In Sect. 5 we show that our results can be used to derive SSD conditions for order statistics of i.i.d. samples from (possibly) different RVs.

\section{Preliminaries}

\subsection{Stochastic orders}

In this paper, we refer to absolutely continuous RVs. Thus, an RV, $U$, has a cumulative distribution function $(\mathrm{CDF}), F_{U}$, a quantile function $(\mathrm{QF}), Q_{U}=F_{U}^{-1}$, and a probability density function $(\mathrm{PDF}), f_{U}$. 
We recall the basic definitions of FSD, SSD and ICX.

1. We say that $U_{1}$ dominates $U_{2}$ w.r.t. FSD and we write $U_{1} \geq_{1} U_{2}$ iff $F_{U_{1}}(u) \leq$ $F_{U_{2}}(u), \forall u \in \mathbb{R}$.

2. We say that $U_{1}$ dominates $U_{2}$ w.r.t. SSD and we write $U_{1} \geq_{2} U_{2}$ iff $\int_{-\infty}^{u} F_{U_{1}}(t) d t \leq$ $\int_{-\infty}^{u} F_{U_{2}}(t) d t, \forall u \in \mathbb{R}$.

3. We say that $U_{2}$ is smaller than $U_{1}$ in the ICX, and we write $U_{1} \geq_{i c x} U_{2}$, iff $\int_{u}^{\infty} 1-F_{U_{1}}(t) d t \geq \int_{u}^{\infty} 1-F_{U_{2}}(t) d t, \forall u \in \mathbb{R}$.

The following relations hold between these stochastic orders [36]:

$$
\begin{aligned}
& U_{1} \geq_{1} U_{2} \Rightarrow U_{1} \geq_{2} U_{2}, U_{1} \geq_{1} U_{2} \Rightarrow U_{1} \geq_{i c x} U_{2}, \\
& U_{1} \geq_{i c x} U_{2} \Longleftrightarrow-U_{2} \geq_{2}-U_{1} .
\end{aligned}
$$

It is apparent that FSD holds iff CDFs do not cross. However, it is interesting to observe that both SSD and ICX can be related to the number of crossings between CDFs or PDFs. In fact, when the integral conditions of the definitions above are difficult to verify for some parametric distributions, we can use an alternative method for deriving sufficient conditions. We denote it as the single-crossing property, because it requires CDFs to cross (at most) once [17]. When it is not possible to verify the crossing condition of CDFs (e.g. when a closed-form expression for the CDF is not available), we rely on some closely related results that involve densities. In particular, it is sufficient to prove that PDFs cross at most twice or that the likelihood ratio (i.e. the ratio of the PDFs) is unimodal $[11,33,35]$.

Let us denote the number of sign changes of a function, $g$, defined on an interval, $I$, with

$$
S^{-}(g)=S^{-}(g(x))=\sup S^{-}\left[g\left(x_{1}\right), \ldots, g\left(x_{m}\right)\right],
$$

where $S^{-}\left[y_{1}, \ldots, y_{m}\right]$ is the number of sign changes of the sequence, $y_{1}, \ldots, y_{m}$, where the zero terms are omitted, and the supremum is extended over all $x_{1}<x_{2}<\ldots<x_{m}\left(x_{i} \in I\right)$, $m<\infty$ [35]. We summarise some important results as follows.

Lemma 1 Let $U_{1}$ and $U_{2}$ have finite means.

1. If $S^{-}\left(F_{U_{1}}-F_{U_{2}}\right) \leq 1$ and the sign sequence starts with - , then $U_{1} \geq_{2} U_{2}$ iff $E\left(U_{1}\right) \geq$ $E\left(U_{2}\right)$, whilst $U_{2} \geq i c x \quad U_{1}$ iff $E\left(U_{2}\right) \geq E\left(U_{1}\right)$.

2. Let $S^{-}\left(f_{U_{1}}-f_{U_{2}}\right) \leq 2$ and the sign sequence begins with - . Then, $U_{1} \geq_{2} U_{2}$ iff $E\left(U_{1}\right) \geq E\left(U_{2}\right)$, whilst $U_{2} \geq_{i c x} U_{1}$ iff $E\left(U_{2}\right) \geq E\left(U_{1}\right)$.

3. Let $f_{U_{1}} / f_{U_{2}}$ be unimodal, where the mode is a supremum. Then, $U_{1} \geq_{2} U_{2}$ iff $E\left(U_{1}\right) \geq$ $E\left(U_{2}\right)$, whilst $U_{2} \geq_{i c x} U_{1}$ iff $E\left(U_{2}\right) \geq E\left(U_{1}\right)$.

Proof Point (1) is Theorem 4.A.22(b) (p. 194) of [36]. Point (2) follows from Lemma 2.1 of [35]. Regarding point (3), the proof can be found in [33], for the SSD case. Then, the ICX condition follows from the relation, $U_{1} \geq_{i c x} U_{2} \Longleftrightarrow-U_{2} \geq_{2}-U_{1}$.

\subsection{The T-X family}

The T-X method, introduced by [5] is based on the composition of the CDFs of two RVs, $X$ and $T$, with a function, say $W$, that fulfils certain requirements. In [2] give a more practical definition by taking $W$ to be the QF of a third RV $Y$. This method, denoted as $T-X\{Y\}$, can be outlined as follows: given three RVs $X, Y$ and $T$, where the support of $T$ coincides with that of $Y$, a new $\mathrm{RV} Z$ is defined by means of the $\mathrm{CDF}$

$$
F_{Z}=F_{T} \circ Q_{Y} \circ F_{X} .
$$


The corresponding PDF is

$$
f_{Z}(z)=f_{X} \frac{f_{T} \circ Q_{Y} \circ F_{X}}{f_{Y} \circ Q_{Y} \circ F_{X}}
$$

Remark 1 Formula (3) has a twofold interpretation:

- The composite function $u=Q_{X} \circ F_{Y}$ can be seen as an increasing transformation of the $\mathrm{RV} T$, since $Z \stackrel{d}{=} u(T)$.

- The composite function $h=F_{T} \circ Q_{Y}$ can be seen as a probability distortion ${ }^{1}$ of the CDF $F_{X}$, since $Z \stackrel{d}{=}[X]_{h}$, where $[X]_{h}$ is the RV with $\mathrm{CDF} h \circ F_{X}$.

These functions will prove useful in the next section.

With the notation introduced by [2] we can specify and highlight the role of the different distributions within the $\mathrm{T}-\mathrm{X}\{\mathrm{Y}\}$ family, e.g. gamma-normal $\{$ exponential $\}$ means that $T$ is a gamma, $X$ is a normal and $Y$ is an exponential. Some basic properties are as follows:

1. $Z$ and $X$ have the same support.

2. If $\theta$ is a location (scale) parameter for $X$, then it is also a location (scale) parameter for $Z$.

3. Any distribution can (trivially) belong to the T-X family (if $F_{Y}=F_{T}$, then $F_{X}=F_{Z}$ ).

4. The $\mathrm{QF}$ of $Z$ is given by $Q_{Z}(t)=Q_{X} \circ F_{Y} \circ Q_{T}(t)$.

5. The expectation of $Z$, if it exists, is given by $E(Z)=\int_{0}^{1} Q_{X} \circ F_{Y} \circ Q_{T}(t) d t$.

Many continuous RVs have closed-form expressions for the QF, which can play the role of $Q_{Y}$ in (3), to generate T-X $\{\mathrm{Y}\}$ families. Since the support of $Y$ coincides with the support of $T$, it is useful to classify different choices of $Y$ on the basis of its support. Among those defined on $(0, \infty)$, we may use the exponential, the Weibull, the Rayleigh, the Dagum, the Lomax, the log-logistic and the exponentiated exponential distribution. Among those with support $(-\infty, \infty)$, we may use the Gumbel, the Laplace, the logistic and the generalised logistic distribution. However, in most practical situations, $F_{Y}$ is not parametrised, since a parametrisation may lead to the collapse of the parameter space.

The original paper of [5] focuses on the $T-X$ \{exponential\} family, which is obtained by taking $Q_{Y}$ to be the $\mathrm{QF}$ of an exponential $\mathrm{RV}$ with scale parameter equal to 1, i.e.: $Q_{Y}(p)=-\ln (1-p)$. The $\mathrm{T}-\mathrm{X}$ \{exponential $\}$ family makes it possible to generate a large number of new families of distributions, such as: the gamma-X family [5] -among which we can find the gamma-normal distribution [6]; the beta-exponential-X family and the Weibull-X family_among which we can find the Weibull-Pareto distribution, as defined by [4]; the exponentiated Marshall-Olkin family [15]; and the Lomax-generator family [14].

Different choices of $Q_{Y}$ make it possible to generate an even larger number of new families, among which we may cite the exponentiated $T$-X family [8], the T-normal family [7], the logistic-X family [38], the Weibull-Pareto, as defined by [3] (not to be confused with the homonymous distribution of [4], the exponentiated Weibull [31] and the new distributions proposed in [2].

Another important advantage of the $\mathrm{T}-\mathrm{X}$ family is that it also generates many existing parametric models of noticeable practical relevance, such as: the GB1, the GB2, the GG [29], or, more generally, the generalised beta of [30] and the generalised beta-generated distribution proposed by [1].

1 A probability distortion function is defined as a non-decreasing function $h$ such that $h(0)=0$ and $h(1)=1$, see [39]. 


\section{Stochastic orders for the T-X-decomposable families}

Because many parametric distributions can be decomposed via $\mathrm{T}-\mathrm{X}$ method, we are concerned with finding the sufficient conditions for ranking T-X families with FSD, SSD (especially), and ICX. We wonder whether T-X families obtained by composition of CDFs and QFs, ranked by FSD, SSD, or ICX, preserve some kind of order. Actually, this is related to the preservation of stochastic orders under transfomations or distortions [25,32].

Below we derive sufficient conditions for FSD, SSD and ICX, for pairs of distributions which are decomposable via the T-X method. Stated otherwise, we compare pairs of distributions with CDFs given by (3).

Henceforth, let:

- $F_{Z_{i}}(z)=F_{T_{i}} \circ Q_{Y_{i}} \circ F_{X_{i}}(z)$, for $i=1,2$;

- $u=Q_{X_{2}} \circ F_{Y_{2}}$

$-h=F_{T_{1}} \circ Q_{Y_{1}}$.

Moreover, denote with $\mathcal{F}_{c v}$ and $\mathcal{F}_{c x}$ the classes of all concave and convex functions, respectively.

Our objective is to compare $Z_{1}$ and $Z_{2}$. As far as concerns the FSD, it is readily seen that

$$
X_{1} \geq_{1} X_{2}, Y_{2} \geq_{1} Y_{1} \text { and } T_{1} \geq_{1} T_{2} \Rightarrow Z_{1} \geq_{1} Z_{2} .
$$

Differently, we propose two approaches for deriving SSD and ICX conditions, based on the classic characterization of stochastic orders in terms of expected utilities [16], and on the dual characterization, based on distorted expectations [39]. The two methods are not mutually exclusive. Theorem 1 has been proved by [26], we provide a simpler proof here.

Theorem $1 u \in \mathcal{F}_{c v}, X_{1} \geq_{1} X_{2}, Y_{2} \geq_{1} Y_{1}$ and $T_{1} \geq_{2} T_{2} \Rightarrow Z_{1} \geq_{2} Z_{2}$.

Proof By characterization of SSD, $T_{1} \geq_{2} T_{2}$ iff $E\left(\phi\left(T_{1}\right)\right) \geq E\left(\phi\left(T_{2}\right)\right)$ for every increasing and concave function $\phi$ [28, Proposition B.19.c]. Consequently, we obtain $E\left(\phi \circ u\left(T_{1}\right)\right) \geq$ $E\left(\phi \circ u\left(T_{2}\right)\right)$ for every increasing concave function $\phi$, that is, $u\left(T_{1}\right) \geq_{2} u\left(T_{2}\right)$.

Now, let $\tilde{u}=Q_{X_{1}} \circ F_{Y_{1}} . X_{1} \geq_{1} X_{2}$ and $Y_{2} \geq_{1} Y_{1}$ yield $\tilde{u}(x) \geq u(x), \forall x \in \mathbb{R}$, then

$$
Z_{1} \stackrel{d}{=} \tilde{u}\left(T_{1}\right) \geq_{1} u\left(T_{1}\right) \geq_{2} u\left(T_{2}\right) \stackrel{d}{=} Z_{2} .
$$

Theorem $2 h \in \mathcal{F}_{c v}, X_{1} \geq_{2} X_{2}, Y_{2} \geq_{1} Y_{1}$ and $T_{1} \geq_{1} T_{2} \Rightarrow Z_{1} \geq_{2} Z_{2}$.

Proof By the dual characterization of SSD, $X_{1} \geq_{2} X_{2}$ iff $E\left(\left[X_{1}\right]_{\psi}\right) \geq E\left(\left[X_{2}\right]_{\psi}\right)$ for every concave distortion function $\psi[25$, Theorem 4]. Consequently, since $h$ is concave by assumption, we obtain $E\left(\left[X_{1}\right]_{\psi \circ h}\right) \geq E\left(\left[X_{2}\right]_{\psi \circ h}\right)$ for every concave distortion function $\psi$, that is, $\left[X_{1}\right]_{h} \geq_{2}\left[X_{2}\right]_{h}$.

Now, let $\tilde{h}=F_{T_{2}} \circ Q_{Y_{2}} . Y_{2} \geq_{1} Y_{1}$ and $T_{1} \geq_{1} T_{2}$ yield $h(p) \leq \tilde{h}(p), \forall p \in[0,1]$, then

$$
Z_{1} \stackrel{d}{=}\left[X_{1}\right]_{h} \geq_{2}\left[X_{2}\right]_{h} \geq_{1}\left[X_{2}\right]_{\tilde{h}} \stackrel{d}{=} Z_{2} .
$$

Remark 2 We observe that, regarding the T-X families studied in the literature, $F_{Y}$ is generally not parametrised. Hence, if $Z_{1}$ and $Z_{2}$ are within the same family, $F_{Y}$ is generally fixed $\left(F_{Y_{1}}=F_{Y_{2}}=F_{Y}\right)$. The previous theorems also apply to this special case, owing to the reflexive relation $Y \geq_{1} Y$. Our results, however, are stated in the most general form to enable comparisons between different families (composed by different $Q_{Y}$ 's). 
Theorems 1 and 2 provide two methods for deriving SSD, in which the first condition depends on the shape of the distribution of $X_{2}$ or $T_{1}$, respectively, whereas the second and the fourth conditions can be obtained just by comparing pairwise $X_{1}$ and $X_{2}$, or $T_{1}$ and $T_{2}$. Counterexamples can show that replacing FSD with SSD in the second condition of Theorem 1, or in the fourth condition of Theorem 2, does not ensure $Z_{1} \geq_{2} Z_{2}$. However, by combining the two methods, we obtain the following:

Theorem $3 u, h \in \mathcal{F}_{c v}, X_{1} \geq_{2} X_{2}, Y_{2} \geq_{1} Y_{1}$ and $T_{1} \geq_{2} T_{2} \Rightarrow Z_{1} \geq_{2} Z_{2}$.

Proof As in the proof of Theorem 1, $u\left(T_{1}\right) \geq_{2} u\left(T_{2}\right) \stackrel{d}{=} Z_{2}$. As in the proof of Theorem 2, $Z_{1} \stackrel{d}{=}\left[X_{1}\right]_{h} \geq_{2}\left[X_{2}\right]_{h}$. Since $u\left(T_{1}\right)$ has CDF $F_{T_{1}} \circ Q_{Y_{2}} \circ F_{X_{2}}$ and $\left[X_{2}\right]_{h}$ has CDF $F_{T_{1}} \circ$ $Q_{Y_{1}} \circ F_{X_{2}}$, the thesis follows from $Y_{2} \geq_{1} Y_{1}$.

Remark 3 If $X_{1}, X_{2}$ belong to the same location-scale family, that is, $F_{X_{i}}(x)=G\left(\frac{x-\mu_{i}}{\sigma_{i}}\right)$, for $i=1,2$, where $G$ is the CDF of the standard RV, say $W$, then $\sigma_{1}<\sigma_{2}$ gives $S^{-}\left(F_{X_{1}}-F_{X_{2}}\right)=$ $S^{-}\left(h \circ F_{X_{1}}-h \circ F_{X_{2}}\right) \leq 1$ (the sign sequence starts with - ) and the crossing point is at

$$
x_{0}=\frac{\mu_{1} \sigma_{2}-\mu_{2} \sigma_{1}}{\sigma_{2}-\sigma_{1}}
$$

(see Lemma 2). Subsequently, by Lemma 1 and $Y_{2} \geq_{1} Y_{1}$, the second condition of Theorem 1 can be replaced by

$$
\sigma_{1}<\sigma_{2} \text { and } \mu_{1}-\mu_{2} \geq E\left([W]_{h}\right)\left(\sigma_{1}-\sigma_{2}\right),
$$

[25, section 3.2], which is indeed sufficient for $Z_{1} \stackrel{d}{=}\left[X_{1}\right]_{h} \geq_{2}\left[X_{2}\right]_{h} \geq_{1} u\left(T_{1}\right)$. Likewise, if $T_{1}, T_{2}$ belong to the same location-scale family, the third condition of Theorem 2 may be replaced by

$$
\sigma_{1}<\sigma_{2} \text { and } \mu_{1}-\mu_{2} \geq u^{-1}\left(\frac{E\left(u\left(\sigma_{1} W\right)\right)}{E\left(u\left(\sigma_{2} W\right)\right)}\right)
$$

[18, section 3.3].

Remark 4 The requirements on $u$ and $h$ impose constraints on the shape of $F_{X_{2}}$ and $F_{T_{1}}$, respectively. Such constraints are related to the concept of relative convexity w.r.t. $F_{Y_{2}}$ and $F_{Y_{1}}$, respectively, which may be also expressed in terms of stochastic orders:

$-u \in \mathcal{F}_{c v}$ iff $F_{Y_{2}} \geq_{c} F_{X_{2}}$, where " $\geq_{c}$ " denotes the convex transform order of [44].

- $h \in \mathcal{F}_{c v}$ iff $F_{Y_{1}} \geq^{c} F_{T_{1}}$, where " $\geq^{c "}$ " denotes the convex order of [13]. ${ }^{2}$

The convex transform order $\geq_{c}$ gives rise to various classes of distributions that are convex w.r.t. simple reference quantile functions, such as the unit exponential quantile $-\ln (1-p)$, the logit function $-\ln \frac{p}{1-p}$ and the odds function $\frac{p}{1-p}$ [27]. The convex order $\geq^{c}$ can be equivalently used to define classes of distributions w.r.t. some simple reference functions. Interestingly, such classes contain most basic models [27] and these simple quantiles are commonly employed for the composition of multiparametric models via the T-X method, as we shall see in the next section. Therefore, theorems 1, 2 and 3 have broad applications. However, in some cases, the proposed methods are not applicable, because, for some T$\mathrm{X}$ families, neither $u$ nor $h$ is concave. A mathematically equivalent approach enables the

$2 \geq_{c}$ is referred to as convex transform order as it requires convexity of the transfomation function $u^{-1}=$ $Q_{Y_{2}} \circ Q_{X_{2}}$. Similarly, $\geq^{c}$ may be referred to as convex distortion order as it requires convexity of the distortion function $h^{-1}=F_{Y_{1}} \circ F_{T_{1}}$. 
derivation of ICX when $u$ or $h$ is convex. We state the following three results in a compact form. The proofs are omitted because they are similar to the previous ones.

\section{Theorem 4}

1. $u \in \mathcal{F}_{c x}, X_{1} \geq_{1} X_{2}, Y_{2} \geq_{1} Y_{1}$ and $T_{1} \geq_{i c x} T_{2} \Rightarrow Z_{1} \geq_{i c x} Z_{2}$;

2. $h \in \mathcal{F}_{c x}, X_{1} \geq_{i c x} X_{2}, Y_{2} \geq_{1} Y_{1}$ and $T_{1} \geq_{1} T_{2} \Rightarrow Z_{1} \geq_{i c x} Z_{2}$;

3. $u, h \in \mathcal{F}_{c x}, X_{1} \geq_{i c x} X_{2}, Y_{2} \geq_{1} Y_{1}$ and $T_{1} \geq_{i c x} T_{2} \Rightarrow Z_{1} \geq_{i c x} Z_{2}$.

\section{Some special cases}

In this section, we apply our method to some special mutliparametric families, i.e., the T$\mathrm{X}$ exponential (among which we derive dominance conditions for the gamma-normal and the Weibull-Pareto\{exponential $\}$ ) and the BG family (among which we derive dominance conditions for the GB1 and the GB2). To apply the method, we need the functions $u$ or $g$ to be concave or convex, so that we can derive sufficient conditions for SSD or ICX, respectively - in general, at least one condition holds true.

\subsection{T-X \{exponential\} family}

The T-X \{exponential \} family, studied by [5], is obtained by setting $Q_{Y}(p)=-\ln (1-p)$. Sufficient SSD conditions for this family are particularly relevant because the assumption of concavity of $u$ is equivalent to the assumption of convexity of the cumulative hazard function of $X_{2}$, namely $-\ln \left(1-F_{X_{2}}\right)$. The class of distributions with convex hazard function is known in the literature as increasing failure rate family (IFR) [27] and contains many important models (i.e. normal, logistic, extreme value, chi-square, chi, exponential, Laplace, Weibull with shape parameter $\geq 1$, gamma with shape parameter $\geq 1$, and beta with both parameters $\geq 1$ [10]). Hence, the method provided by Theorem 1 has broad application within this family. Conversely, both Pareto (I) and Pareto (II) have concave hazard function (i.e., $u$ is convex), which is know in the literature as having a decreasing failure rate (DFR).

\subsubsection{Gamma-normal distribution}

The gamma-normal \{exponential \} distribution, introduced and studied by [6] is obtained by taking $T$ as a gamma, $X$ as a normal, and $Y$ as an exponential. This distribution always has a finite mean. Neither the gamma nor the normal distribution has a closed-form expression for the CDF:

$$
\begin{gathered}
F_{T}(t ; a, b)=\gamma\left[a, \frac{t}{b}\right] / \Gamma(a), a, b, t>0 ; \\
F_{X}(x ; \mu, \sigma)=\Phi\left(\frac{\mu-x}{\sigma}\right), \sigma>0 ; x, \mu \in \mathbb{R},
\end{gathered}
$$

where $\gamma$ is the (lower) incomplete gamma function and $\Phi$ is the CDF of the standard normal. Note that $a$ is a shape parameter, $b$ and $\sigma$ are scale parameters and $\mu$ is a location parameter.

Then, the CDF of $Z$ is given by

$$
F_{Z}(z ; a, b, \mu, \sigma)=\gamma\left[a,-\ln \left(1-\Phi\left(\frac{\mu-z}{\sigma}\right)\right) / b\right] / \Gamma(a), z \in \mathbb{R} .
$$

In this expression, $b$ is an additional shape parameter. Let $Z_{i}$, for $i=1,2$, have a gamma-normal distribution, $F_{Z_{i}}(z)=F_{Z}\left(z ; a_{i}, b_{i}, \mu_{i}, \sigma_{i}\right)$, where $F_{T_{i}}(z)=F_{T}\left(t ; a_{i}, b_{i}\right)$ 
and $F_{X_{i}}(z)=F_{X}\left(x ; \mu_{i}, \sigma_{i}\right)$. We search for sufficient conditions for $Z_{1} \geq_{2} Z_{2}$. We cannot apply Lemma 1 (point 1 ), because $F_{Z}$ is not in closed form. Thus, it is not possible to directly verify the single-crossing condition on $F_{Z_{1}}, F_{Z_{2}}$. Moreover, we cannot use the double-crossing argument of [35] (Lemma 1 point 2) nor the unimodality condition (Lemma 1 point 3 ), to be verified with PDFs, because $f_{Z}$ does not have a closed form as well. Thus, in this case, the simplest solution is to rely on the dominance conditions for the normal and the gamma, which are already known and easy to derive.

The normal distribution is IFR, that is, $u$ is concave, therefore we can apply Theorem 1 by relying on FSD conditions for the normal and SSD conditions for the gamma [33]. We obtain that

$$
\mu_{1}=\mu_{2}, \sigma_{1} \leq \sigma_{2}, a_{1} \geq a_{2} \text { and } a_{1} b_{1} \geq a_{2} b_{2} \Rightarrow Z_{1} \geq_{2} Z_{2} .
$$

Note that $F_{Y_{1}} \circ Q_{T_{1}}$ is convex, that is, $h$ is concave, iff the likelihood ratio

$$
\frac{f_{Y_{1}}}{f_{T_{1}}}(x)=\Gamma(a) b^{a} x^{1-a} e^{\frac{(1-b) x}{b}}
$$

is increasing [13]. Then $h$ is concave for $a_{1}, b_{1} \leq 1$ and Theorem 3 gives

$$
\mu_{1} \geq \mu_{2}, \sigma_{1} \leq \sigma_{2}, 1 \geq a_{1} \geq a_{2}, b_{1} \leq 1 \text { and } a_{1} b_{1} \geq a_{2} b_{2} \Rightarrow Z_{1} \geq_{2} Z_{2} .
$$

\subsubsection{Weibull-Pareto \{exponential\}}

The Weibull-Pareto\{exponential \} distribution, as introduced and studied by [4], is obtained by taking $T$ as a two-parameter Weibull distribution with $\operatorname{PDF} F_{T}(t ; c, \gamma)=1-$ $\exp \left[-\left(\frac{t}{\gamma}\right)^{c}\right], c, \gamma>0, t>0, X$ as a Pareto (I) distribution with $\mathrm{CDF} F_{X}(x ; k, \theta)=$ $1-\left(\frac{\theta}{x}\right)^{k}, k>0, x>\theta>0$ and $Y$ as an exponential distribution with parameter 1 . The CDF of $Z$ reduces to

$$
F_{Z}(z ; c, \beta, \theta)=1-e^{-\left(\beta \log \left(\frac{z}{\theta}\right)\right)^{c}}, z>\theta>0
$$

where $\beta=\frac{k}{\gamma}$. This family is indistinguishable with that obtained under the assumption that $X$ follows a Pareto (I) distribution with CDF $F_{X}(x, 1, \theta)$. Then, let $Z_{i}$, for $i=1,2$, have a Weibull-Pareto $\{$ exponential $\}$ distribution $F_{Z_{i}}(z)=F_{Z}\left(z, c_{i}, \gamma_{i}, \theta_{i}\right)$, where $F_{T_{i}}(z)=$ $F_{T}\left(t ; c_{i}, \gamma_{i}\right)$ and $F_{X_{i}}(x)=F_{X}\left(x ; 1, \theta_{i}\right)$. As noted above, the Pareto (I) is DFR and ICX can be derived straightforwardly. Indeed, it can be shown that $T_{1} \geq_{i c x} T_{2}$ if $c_{1} \leq c_{2}$ and $\gamma_{1} \Gamma\left(1+1 / c_{1}\right) \geq \gamma_{2} \Gamma\left(1+1 / c_{2}\right)$ [37], whereas $X_{1} \geq_{1} X_{2}$ iff $\theta_{1} \geq \theta_{2}$, and we obtain that:

$$
c_{2} \geq c_{1}, \gamma_{1} \Gamma\left(1+\frac{1}{c_{1}}\right) \geq \gamma_{2} \Gamma\left(1+\frac{1}{c_{2}}\right) \text { and } \theta_{1} \geq \theta_{2} \Rightarrow Z_{1} \geq_{i c x} Z_{2} .
$$

\subsection{Beta-generated family}

The BG family has been introduced by [19]. Besides generating the GB1 and GB2, the BG is very important because of its relation with order statistics, as we shall discuss in the sequel. Starting from a baseline RV $X$, the BG is defined by the following CDF

$$
F_{B}\left(F_{X}(z) ; p, q\right)
$$

where $F_{B}(x ; p, q)$ is the beta $\mathrm{CDF}$ and $z$ is such that $f_{X}(z)>0$. 
The BG generates the two main distributions for modelling size phenomena on bounded or unbounded support, namely, the GB1 and the GB2, respectively. Many other widely used models can be obtained from the GB1 and the GB2, such as the standard beta, power function, the Singh-Maddala, the Dagum or Burr (III), the B2, the log-logistic, and the Pareto (II). The generalised gamma (also the gamma and the Weibull) can be seen as a limiting case of the GB2 [22].

The BG model can be decomposed by using the beta distribution as the generator $T$ and the uniform for the QF, thereby giving rise to the beta-X \{uniform\} family. However, since the $\mathrm{QF}$ of the uniform distribution is the identity function, to apply Theorem 1 we would require the convexity of $u^{-1}=Q_{Y} \circ F_{X}=F_{X}$, which is a highly restrictive condition for a CDF. Thus, we use an alternative $\mathrm{T}-\mathrm{X}\{\mathrm{Y}\}$ decomposition, thereby rendering it possible to consider the $\mathrm{BG}$ as a $\mathrm{B} 2-\mathrm{X}\{\log$-logistic $\}$ family. In particular, the $\mathrm{B} 2$ has $\mathrm{CDF} F_{B}\left(\frac{x}{x+1} ; p, q\right)$, where $\frac{x}{x+1}=F_{Y}(x)$ is in fact the CDF of a log-logistic distributions with both parameters equal to 1 (consequently, $Q_{Y}(p)=\frac{p}{1-p}$ ). We show that SSD conditions within the BG family can be derived easily if $u^{-1}=\frac{F_{X}}{1-F_{X}}$, namely, the odds function of $F_{X}$, is convex. Most basic models fulfil this condition, namely all IFR distributions plus some heavy tailed ones [27]. Similarly, ICX conditions can be derived by requiring the odds function to be concave (yet, this condition is quite strong). Moreover, $h$ is actually the beta CDF, which is concave for $p_{1} \leq 1$ and $q_{1} \geq 1$ (the latter condition ensures also that the $\mathrm{B} 2$ has finite mean). Therefore, we have the following results (the SSD conditions for the B2 are derived in the Appendix; point 1) was proved by [26]):

Corollary 1 For $i=1,2$, let $F_{Z_{i}}=F_{B}\left(F_{X_{i}} ; p_{i}, q_{i}\right)$ and $\frac{F_{X_{2}}}{1-F_{X_{2}}} \in \mathcal{F}_{c x}$.

1. $X_{1} \geq_{1} X_{2}, p_{1} \geq p_{2}$ and $\frac{p_{1}}{q_{1}-1} \geq \frac{p_{2}}{q_{2}-1}\left(q_{1} \geq 1\right) \Rightarrow Z_{1} \geq_{2} Z_{2}$.

2. $X_{1} \geq_{2} X_{2}, 1 \geq p_{1} \geq p_{2}$ and $\frac{p_{1}}{q_{1}-1} \geq \frac{p_{2}}{q_{2}-1}\left(q_{1} \geq 1\right) \Rightarrow Z_{1} \geq_{2} Z_{2}$.

SSD conditions for the GB1 and the GB2 can be derived as follows.

\subsubsection{GB1 distribution}

The GB1, introduced by [29], is suitable to model size phenomena on a bounded support. The GB1 yields the standard beta and the power function distributions as special cases: the two models used to compose the GB1 with the T-X method. Stochastic orders for the GB1 have been studied by [41], who provided four sets of sufficient conditions for the LO. It appears that stronger results are unavailable at present.

The GB1 can be seen as a B2-power function $\{\log$-logistic $\}$ family: $T$ has a B2 distribution with parameters $p, q>0, X$ has a power function distribution with $\mathrm{CDF} F_{X}(x ; a, b)=$ $(x / b)^{a}$ on its support $(0, b)$, where $a, b>0$, and $Q_{Y}(p)=p /(1-p)$. Hence, the T-X method yields the PDF of the GB1:

$$
f_{Z}(z ; p, q, a, b)=\frac{a z^{a p-1}\left(1-(z / b)^{a}\right)^{q-1}}{b^{a p} B(p, q)}, 0<z<b .
$$

Let $Z_{i}$, for $i=1,2$, have a GB1 distribution $F_{Z_{i}}(z)=F_{Z}\left(z ; p_{i}, q_{i}, a_{i}, b_{i}\right)$, where $F_{T_{i}}(z)=$ $F_{T}\left(t ; p_{i}, q_{i}\right)$ and $F_{X_{i}}(z)=F_{X}\left(x ; a_{i}, b_{i}\right)$. It is easy to see that $a_{1} \geq a_{2}$ and $b_{1} \geq b_{2}$ imply $X_{1} \geq_{1} X_{2}$ (Lemma 2). $\frac{F_{X}}{1-F_{X}}$ is convex $\forall a, b>0$. Thus, Corollary 1 point 1 ) yields

$$
a_{1} \geq a_{2}, b_{1} \geq b_{2}, p_{1} \geq p_{2} \text { and } \frac{p_{1}}{q_{1}-1} \geq \frac{p_{2}}{q_{2}-1} \Rightarrow Z_{1} \geq_{2} Z_{2} \text {. }
$$


Note that $a_{1} \geq a_{2}$ implies $S^{-}\left(F_{X_{1}}-F_{X_{2}}\right) \leq 1$ (where the sign sequence starts with - ). Then, since the mean of the power function distribution is $\frac{b}{a+1}$, Corollary 1 point 2) yields

$$
\begin{aligned}
& a_{1} \geq a_{2}, \frac{b_{1}}{a_{1}+1} \geq \frac{b_{2}}{a_{2}+1}, \\
& 1 \geq p_{1} \geq p_{2} \text { and } \frac{p_{1}}{q_{1}-1} \geq \frac{p_{2}}{q_{2}-1} \Rightarrow Z_{1} \geq_{2} Z_{2} .
\end{aligned}
$$

\subsubsection{GB2 distribution}

The GB2, introduced by [29], is probably the main distribution for modelling size phenomena on the unbounded support $(0, \infty)$, owing to its flexibility. Many other widely used models can be obtained from the GB2, such as the Singh-Maddala, the Burr (III), the B2, the loglogistic, and the Pareto (II). The generalised gamma (also the gamma and the Weibull) can be seen as a limiting case of the GB2 [22]. Sufficient conditions for the LO for the GB2 were obtained by [40] and extended by [21].

The GB2 can be obtained as a T-X $\{\mathrm{Y}\}$ family, particularly as a B2-log-logistic $\{$ loglogistic \} family. $T$ has a B2 distribution with parameters $p, q>0, X$ has a log-logistic distribution with $\operatorname{CDF}_{X}(x ; a, b)=\frac{1}{(b / x)^{a}+1}$, where $a, b, x>0$, and $Q_{Y}(p)=p /(1-p)$. Thus, the T-X method yields the PDF of the GB2:

$$
f_{Z}(z ; p, q, a, b)=\frac{a z^{a p-1}}{b^{a p} B(p, q)\left(1+(z / b)^{a}\right)^{p+q}}, z>0 .
$$

Note that the GB2 has a finite mean only if $a q>1$. Let $Z_{i}$, for $i=1,2$, have a GB2 distribution, where $F_{T_{i}}(z)=F_{T}\left(t ; p_{i}, q_{i}\right)$ and $F_{X_{i}}(z)=F_{X}\left(x ; a_{i}, b_{i}\right)$. By applying our method, we find that $X_{1} \geq_{1} X_{2}$ if $b_{1} \geq b_{2}$ and $a_{1}=a_{2}$ (Lemma 2). Moreover $\frac{F_{X}(x)}{1-F_{X}(x)}=$ $(x / b)^{a}$ is convex for $a \geq 1$. Let $F_{\tilde{Z}_{1}}(z)=F_{Z}\left(z ; p_{1}, q_{1}, a_{2}, b_{1}\right)$. Corollary 1 part 1$)$ yields that, if $a_{1}=a_{2} \geq 1, b_{1} \geq b_{2}, p_{1} \geq p_{2}$ and $\frac{p_{1}}{q_{1}-1} \geq \frac{p_{2}}{q_{2}-1}$, then $\tilde{Z}_{1} \geq_{2} Z_{2}$. Next, the conditions obtained can be easily extended, because it is easy to verify that $E\left(\tilde{Z}_{1}\right) \leq E\left(Z_{1}\right)$ and that the ratio, $f_{Z_{1}} / f_{\tilde{Z}_{1}}$, is unimodal. Thus, we can apply Lemma 1 (Point 3). Therefore, we obtain that $Z_{1} \geq_{2} \tilde{Z}_{1} \geq_{2} Z_{2}$, which implies that the sufficient conditions for SSD, can be expressed as follows.

$$
a_{2} \geq a_{1} \geq 1, b_{1} \geq b_{2}, p_{1} \geq p_{2} \text { and } \frac{p_{1}}{q_{1}-1} \geq \frac{p_{2}}{q_{2}-1} \Rightarrow Z_{1} \geq_{2} Z_{2} .
$$

Similarly, Corollary 1 part 2) gives

$$
\begin{aligned}
& a_{2} \geq a_{1} \geq 1, \frac{\pi b_{1} \csc \left(\frac{\pi}{a_{1}}\right)}{a_{1}} \geq \frac{\pi b_{2} \csc \left(\frac{\pi}{a_{2}}\right)}{a_{2}}, \\
& 1 \geq p_{1} \geq p_{2} \text { and } \frac{p_{1}}{q_{1}-1} \geq \frac{p_{2}}{q_{2}-1} \Rightarrow Z_{1} \geq_{2} Z_{2},
\end{aligned}
$$

where the second inequality involves the means of the log-logistic RVs.

\section{An application to order statistics}

Let $X_{1}, \ldots, X_{n}$ denote a sample of i.i.d. RVs from an RV $X$. The $k$-th order statistic is denoted by $X_{k: n}$. It can be shown that the distributions of order statistics of i.i.d. samples 
from any underlying distribution $X$ belong to the BG class. This enables the comparison of order statistics in terms of SSD in various sampling scenarios. In reliability theory, stochastic comparisons of order statistics are particularly relevant. Order statistics may represent the waiting time until fewer than $k$ components remain functioning in a system of $n$ components. Put otherwise, the lifetime of the system is represented as $X_{k: n}$. Thus, reliability engineering is concerned with maximizing the mean life while also reducing the variability since predictable life length is desirable. In the literature, several works deal with this issue using the LO $[9,23,24,42]$; however, we argue that this scenario is even more suitable for SSD, in that it considers both the variability and the mean (the LO is a size-independent version of SSD for non-negative RVs).

As well known, the order statistic $X_{k: n}$ belongs to the BG family. Replacing $p=k$ and $q=n-k+1$ in (18), the CDF of $X_{k: n}$ is

$$
F_{B}\left(F_{X} ; k, n-k+1\right)
$$

Let $X_{1}, \ldots, X_{n}$ denote a sample of i.i.d. RVs from an RV $X$ and let $Y_{1}, \ldots, Y_{m}$ denote a sample of i.i.d. RVs from another RV $Y$. Corollary 1 enables the determination of the sample sizes $n$ and $m$ and the ranks $i$ and $j$ such that $X_{i: n} \geq_{2} Y_{j: m}$. Given $j, n$ and $m$, we can determine the minimum rank $i=i(j, n, m)$ such that $X_{i: n} \geq_{2} Y_{j: m}$. Given $i, j$, and $m$, we can determine minimum the sample size $n=n(i, j, m)$ such that $X_{i: n} \geq_{2} Y_{j: m}$.

Let $X$ and $Y$ be RVs such that $\frac{F_{Y}}{1-F_{Y}}$ is convex. Corollary 1 part 1) yields

$$
X \geq_{1} Y, i \geq j \text { and } \frac{i}{n} \geq \frac{j}{m} \Rightarrow X_{i: n} \geq_{2} Y_{j: m}
$$

(see also [26]), whereas part 2) gives the following result involving sample minima

$$
X \geq_{2} Y \text { and } n \leq m \Rightarrow X_{1: n} \geq_{2} X_{1: m} .
$$

If $X$ and $Y$ have finite means, then $X_{i: n}$ and $Y_{j: m}$ also have finite means (by the Jensen's inequality). In this case, the condition generated by the means of the B2 (i.e., $\frac{i}{n} \geq \frac{j}{m}$ ) can be replaced by $E\left(X_{i: n}\right) \geq E\left(Y_{j: m}\right)$, whereas $i \geq j$ and $X \geq_{2} Y$ are enough to ensure that the CDF of $X_{i: n}$ crosses that of $Y_{j: m}$ at most once from below. Hence, we obtain the following alternative result

$$
X \geq_{2} Y, i \geq j \text { and } E\left(X_{i: n}\right) \geq E\left(Y_{j: m}\right) \Rightarrow X_{i: n} \geq_{2} Y_{j: m} .
$$

Condition (28) is weaker that (26), if the components' distributions are know. Nevertheless, the main advantage of condition (26) is that it's distribution-free, since the only distributional assumption about regards the convexity of $\frac{F_{Y}}{1-F_{Y}}$. These results can be easily applied to several basic models.

Example 1 A basic issue in engineering is designing a system with the smallest variability, subject to given mean life constraints. An $n$-component system that fails if and only if at least $k$ of the $n$ components fail is called a $k$-out-of- $n$ : $F$ system. If the components of such system are i.i.d., its lifetime is represented as $X_{k: n}$. Consider a $j$-out-of- $m$ : F system, with $m=30$ components, say $Y_{1} \ldots Y_{m}$ which fails iff at least $j=10$ components fail. Suppose we need to replace the system with a new one, say $X_{1}, \ldots, X_{n}$, with a larger number of components $n>m$. We want the new system to fail iff at least $i=15$ components fail. What is the largest possible number of components, say $n^{*}$, such that the lifetime of the new system SSD-dominates the old one? If the components of the new system have the same distribution of the old one, then $n^{*}=15 \cdot 30 / 10=45$. Thus, $X_{15: 45} \geq_{2} Y_{10: 30}$, 

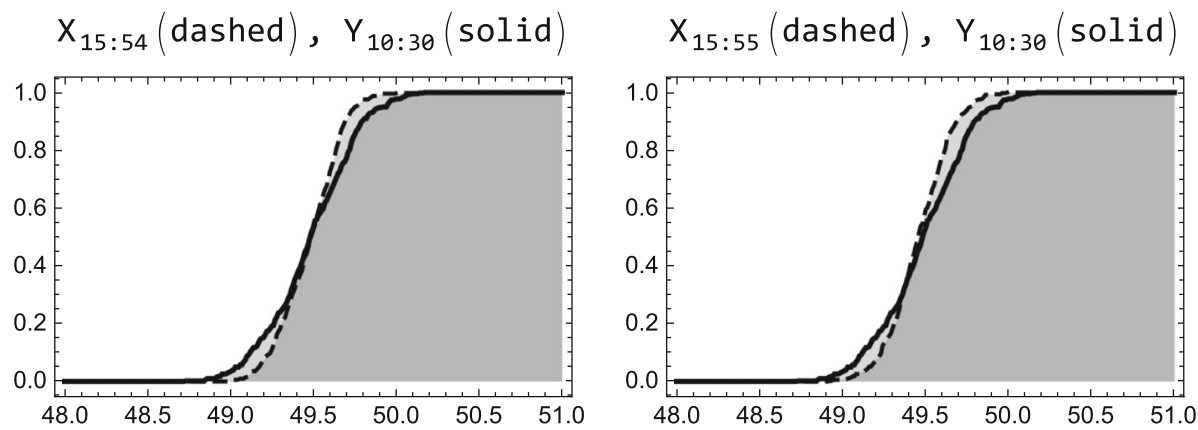

Fig. 1 Empirical CFDs of $X_{15: 55}$ vs. $Y_{10: 30}$ and $X_{15: 54}$ vs. $Y_{10: 30}$

which is quite logical. This result does not rely on any assumption on the mathematical form of the components' distribution. But, the ordering conditions can be weakened if the components' distribution is known. Assume that $X_{1}, \ldots, X_{n}$ are normally distributed with mean $\mu_{X}=50.1$ and standard deviation $\sigma_{X}=1$, whereas $Y_{1}, \ldots, Y_{n}$ are normally distributed with mean $\mu_{X}=50$ and standard deviation $\sigma_{X}=1$. Here the condition $\frac{i}{n} \geq \frac{j}{m}$ can be replaced by $E\left(X_{15: n}\right) \geq E\left(Y_{10: 30}\right)$, where numerical computation yields $n^{*}=54$. Then, in this case we have $X_{15: 54} \geq_{2} Y_{10: 30}$, that is, the number of components can be increased from 45 to 54 . This theoretical result may be supported by a simulation study. We generated 500 random samples from $X$ and $Y$ of sizes $n=54,55$ and $m=30$, respectively, to yield 500 random observations of $X_{(15: 53)}, X_{(15: 54)}$ and $Y_{(10: 30)}$. The (empirical) CDFs of $X_{15: 55}$ vs. $Y_{10: 30}$ and $X_{15: 54}$ vs. $Y_{10: 30}$ are plotted in Fig. 1. Denote by $U^{o b s}$ an RV that is determined by the empirical distribution of a sample from an RV $U$. We observed $X_{15: 54}^{o b s} \geq_{2} Y_{10: 30}^{o b s}$ (singlecrossing CDFs with $\left.E\left(X_{15: 54}^{o b s}\right)=49.49>49.48=E\left(Y_{10: 30}^{o b s}\right)\right)$ whereas $X_{15: 55}^{o b s} \geq_{2} Y_{10: 30}^{o b s}$ does not hold (single-crossing CDFs with $E\left(X_{15: 55}^{o b s}\right)=49.47<49.48=E\left(Y_{10: 30}^{o b s}\right)$ ).

\section{Conclusion}

The approach proposed in this paper provides rules (which may be verifiable with reasonable simplicity) to establish stochastic rankings with FSD, SSD or ICX, for a large number of multi-parametric families of distributions, namely, all the those that can be decomposed via the $\mathrm{T}-\mathrm{X}$ technique. The method has a wide range of applications. Overall, most generalised families of distributions, including the distributions of some classic statistical tools, such as order statistics, and classic transformations of random variables (RV) through invertible functions, can be seen as a composition of parametric functions and can be framed within this approach. Further applications and extensions of our results may be (i) obtaining rules for stochastic dominance between different $\mathrm{T}-\mathrm{X}$ families; (ii) focusing on different stochastic orders; and (iii) deriving further properties for order statistics.

Acknowledgements We are grateful to an anonymous referee for providing insightful comments on a previous version of this manuscript.

Funding T.L. was supported by the Czech Science Foundation (GACR) under project 20-16764S and moreover by SP2020/11, an SGS research project of VŠB-TU Ostrava. The support is greatly acknowledged. 
Funding Open access funding provided by Università degli Studi di Bergamo within the CRUI-CARE Agreement.

Open Access This article is licensed under a Creative Commons Attribution 4.0 International License, which permits use, sharing, adaptation, distribution and reproduction in any medium or format, as long as you give appropriate credit to the original author(s) and the source, provide a link to the Creative Commons licence, and indicate if changes were made. The images or other third party material in this article are included in the article's Creative Commons licence, unless indicated otherwise in a credit line to the material. If material is not included in the article's Creative Commons licence and your intended use is not permitted by statutory regulation or exceeds the permitted use, you will need to obtain permission directly from the copyright holder. To view a copy of this licence, visit http://creativecommons.org/licenses/by/4.0/.

\section{A Location-scale families}

Lemma 2 1. Let $X_{i}$, for $i=1,2$, be RVs with location parameters $\mu_{i}$, such that $F_{X_{i}}(z)=$ $G\left(z-\mu_{i}\right)$. If $\mu_{1} \geq \mu_{2}$, then, $X_{1} \geq_{1} X_{2}$.

2. Let $U_{i}$, for $i=1,2$, be non-negative $R V s$ with scale parameters $\sigma_{i}$, such that $F_{X_{i}}(x)=$ $G\left(x / \sigma_{i}\right)$. If $\sigma_{1} \geq \sigma_{2}$, then $X_{1} \geq_{1} X_{2}$.

3. Let $X_{i}$, for $i=1,2$, be two RVs with location and scale parameters $\mu_{i}$ and $\sigma_{i}$, such that $F_{X_{i}}(x)=G\left(\left(x-\mu_{i}\right) / \sigma_{i}\right)$. If $X_{1}$ and $X_{2}$ have finite means and $\sigma_{2} \geq \sigma_{1}$, then $E\left(X_{1}\right) \geq E\left(X_{2}\right)$ implies $X_{1} \geq_{2} X_{2}$, whereas $E\left(X_{2}\right) \geq E\left(X_{1}\right)$ implies $X_{2} \geq_{i c x} X_{1}$.

Proof Points (1) and (2) can be verified in a straightforward manner.

(3) $G\left(\left(x-\mu_{1}\right) / \sigma_{1}\right)=G\left(\left(x-\mu_{2}\right) / \sigma_{2}\right)$ iff $\left(z-\mu_{1}\right) / \sigma_{1}=\left(z-\mu_{2}\right) / \sigma_{2}$, that holds iff $x=\frac{\mu_{1} \sigma_{2}-\mu_{2} \sigma_{1}}{\sigma_{2}-\sigma_{1}}$. Thus, $S^{-}\left(F_{X_{1}}-F_{X_{2}}\right) \leq 1$, where, in case of equality, the sign sequence starts with - , and point 1$)$ of Lemma 1 yields the thesis.

\section{B On sufficient dominance conditions fot the B2 distribution}

Let $X_{1}$ and $X_{2}$ be two B2-distributed RVs with PDFs $f_{i}(x)=B\left(p_{i}, q_{i}\right)^{-1} x^{p_{i}-1}(1+$ $x)^{-p_{i}-q_{i}}$, where $x, p_{i}, q_{i}>0, i=1,2$. We find $h(x)=\frac{f_{1}(x)}{f_{2}(x)}=a(x)(c+d x)$, where $a(x)>0$ for every $x, p_{i}, q_{i}>0, i=1,2, c=p_{1}-p_{2}$ and $d=q_{2}-q_{1}$. If $c=0$ and $d=0$ the two distributions coincide. Otherwise, we find that, 1) if $c d \geq 0$, then $h$ is a (strictly) monotone function, whereas 2) if $c d<0$, then $h$ is unimodal. We distinguish these two cases.

(1) By virtue of the monotonicity, if $c d \geq 0$, then $S^{-}\left(f_{1}-f_{2}\right)=1$. Then $X_{1}$ and $X_{2}$ are stochastically ordered (Shaked, 1982). More precisely, if $p_{1} \geq p_{2}$ and $q_{2} \geq q_{1}$, then $X_{1} \geq_{1} X_{2}$, because $h$ is increasing.

(2) By virtue of the unimodality of $h$, if $c d<0$, then $S^{-}\left(f_{1}-f_{2}\right) \leq 2$. More precisely, if $p_{1}>p_{2}$ and $q_{1}>q_{2}$, then the mode is a maximum. Thus, applying Lemma 1 (point 3), and, recalling that $E\left(X_{i}\right)=\frac{p_{i}}{q_{i}-1}$, if $q_{i}>1$, we obtain:

1. $p_{1}>p_{2}, q_{1}>q_{2}>1$ and $\frac{p_{1}}{q_{1}-1} \geq \frac{p_{2}}{q_{2}-1}$ implies $X_{1} \geq_{2} X_{2}$.

2. $p_{1}>p_{2}, q_{1}>q_{2}>1$ and $\frac{p_{1}}{q_{1}-1} \leq \frac{p_{2}}{q_{2}-1}$ implies $X_{2} \geq_{i c x} X_{1}$.

Because $X_{1} \geq_{1} X_{2}$ implies $X_{1} \geq_{2} X_{2}$, we may determine a single sufficient condition for SSD: $p_{1} \geq p_{2}$ and $E\left(X_{1}\right) \geq E\left(X_{2}\right)$, if the expectations exist, implying $X_{1} \geq_{2} X_{2}$. 


\section{References}

1. Alexander, C., Cordeiro, G.M., Ortega, E.M., Sarabia, J.M.: Generalized beta-generated distributions. Comput. Stat. Data Anal. 56(6), 1880-1897 (2012)

2. Aljarrah, M.A., Lee, C., Famoye, F.: On generating TX family of distributions using quantile functions. J. Stat. Distrib. Appl. 1(1), 2 (2014)

3. Aljarrah, M.A., Famoye, F., Lee, C.: A new Weibull-Pareto distribution. Commun. Stat. Theory Methods 44(19), 4077-4095 (2015)

4. Alzaatreh, A., Famoye, F., Lee, C.: Weibull-Pareto distribution and its applications. Commun. Stat. Theory Methods 42(9), 1673-1691 (2013a)

5. Alzaatreh, A., Lee, C., Famoye, F.: A new method for generating families of continuous distributions. Metron 71(1), 63-79 (2013b)

6. Alzaatreh, A., Famoye, F., Lee, C.: The gamma-normal distribution: Properties and applications. Comput. Stat. Data Anal. 69, 67-80 (2014a)

7. Alzaatreh, A., Lee, C., Famoye, F.: T-normal family of distributions: a new approach to generalize the normal distribution. J. Stat. Distrib. Appl. 1(1), 16 (2014b)

8. Alzaghal, A., Famoye, F., Lee, C.: Exponentiated $t-x$ family of distributions with some applications. Int. J. Stat. Prob. 2(3), 31 (2013)

9. Arnold, B.C., Villaseñor, J.A.: Lorenz ordering of order statistics. Stochastic orders and decision under risk IMS Lecture Notes-Monograph Series 38-47 (1991)

10. Bagnoli, M., Bergstrom, T.: Log-concave probability and its applications. Econ. Theor. 26(2), 445-469 (2005)

11. Belzunce, F., Martinez-Riquelme, C., Ruiz, J.M.: On sufficient conditions for mean residual life and related orders. Comput. Stat. Data Anal. 61, 199-210 (2013a)

12. Belzunce, F., Pinar, J.F., Ruiz, J.M., Sordo, M.A.: Comparison of concentration for several families of income distributions. Stat. Prob. Lett. 83(4), 1036-1045 (2013b)

13. Chan, W., Proschan, F., Sethuraman, J.: Convex-ordering among functions, with applications to reliability and mathematical statistics. Lecture Notes-Monograph Series 121-134 (1990)

14. Cordeiro, G.M., Ortega, E.M., Popović, B.V., Pescim, R.R.: The Lomax generator of distributions: Properties, minification process and regression model. Appl. Math. Comput. 247, 465-486 (2014)

15. Dias, C.R., Cordeiro, G.M., Alizadeh, M., Marinho, P.R.D., Coêlho, H.F.C.: Exponentiated MarshallOlkin family of distributions. J. Stat. Distrib. Appl. 3(1), 15 (2016)

16. Fishburn, P.C.: Stochastic dominance and moments of distributions. Math. Oper. Res. 5(1), 94-100 (1980)

17. Hanoch, G., Levy, H.: The efficiency analysis of choices involving risk. Rev. Econ. Stud. 36(3), 335-346 (1969)

18. Huang, R.J., Tzeng, L.Y., Zhao, L.: Fractional degree stochastic dominance. Management Science (2020)

19. Jones, M.: Families of distributions arising from distributions of order statistics. Test 13(1), 1-43 (2004)

20. Karlin, S., Novikoff, A.: Generalized convex inequalities. Pac. J. Math. 13(4), 1251-1279 (1963)

21. Kleiber, C.: On the Lorenz order within parametric families of income distributions. Sankhyā: Indian J. Stat. Series B 514-517 (1999)

22. Kleiber, C., Kotz, S.: Statistical size distributions in economics and actuarial sciences. Wiley Series in Probability and Statistics (2003)

23. Kochar, S.: Lorenz ordering of order statistics. Stat. Prob. Lett. 76(17), 1855-1860 (2006)

24. Kochar, S.: Stochastic comparisons of order statistics and spacings: a review. ISRN Probability and Statistics 2012, (2012)

25. Lando, T., Bertoli-Barsotti, L.: Distorted stochastic dominance: a generalized family of stochastic orders. J. Math. Econ. 90, 132-139 (2020). https://doi.org/10.1016/j.jmateco.2020.07.005

26. Lando, T., Bertoli-Barsotti, L.: Second-order stochastic dominance for decomposable multiparametric families with applications to order statistics. Stat. Prob. Lett. 159, 108691 (2020)

27. Marshall, A.W., Olkin, I.: Life distributions, vol. 13. Springer (2007)

28. Marshall, A.W., Olkin, I., Arnold, B.C.: Inequalities: theory of majorization and its applications. Springer (2011)

29. McDonald, J.B.: Some generalized functions for the size distribution of income. Econometrica: journal of the Econometric Society 647-663 (1984)

30. McDonald, J.B., Xu, Y.J.: A generalization of the beta distribution with applications. J. Econ. 66(1-2), 133-152 (1995)

31. Nadarajah, S., Cordeiro, G.M., Ortega, E.M.: The exponentiated Weibull distribution: a survey. Stat. Pap. 54(3), 839-877 (2013) 
32. Navarro, J., del Águila, Y., Sordo, M.A., Suárez-Llorens, A.: Stochastic ordering properties for systems with dependent identically distributed components. Appl. Stochas. Models Bus. Ind. 29(3), 264-278 (2013)

33. Ramos, H.M., Ollero, J., Sordo, M.A.: A sufficient condition for generalized Lorenz order. J. Econ. Theory 90(2), 286-292 (2000)

34. Sarabia, J.M., Castillo, E., Slottje, D.J.: Lorenz ordering between McDonald's generalized functions of the income size distribution. Econ. Lett. 75(2), 265-270 (2002)

35. Shaked, M.: Dispersive ordering of distributions. J. Appl. Prob. 19(2), 310-320 (1982)

36. Shaked, M., Shanthikumar, J.G.: Stochastic orders. Springer Series in Statistics (2007)

37. Stoyan, D.: Comparison methods for queues and other stochastic models. 1983. John Wiley\&Sons (1983)

38. Tahir, M., Cordeiro, G.M., Alzaatreh, A., Mansoor, M., Zubair, M.: The logistic-X family of distributions and its applications. Commun. Stat. Theory Methods 45(24), 7326-7349 (2016)

39. Wang, S.S., Young, V.R.: Ordering risks: Expected utility theory versus Yaari's dual theory of risk. Insur. Math. Econ. 22(2), 145-161 (1998)

40. Wilfling, B.: Lorenz ordering of generalized beta-ii income distributions. J. Econ. 71(1-2), 381-388 (1996a)

41. Wilfling, B.: Lorenz ordering of power-function order statistics. Stat. Prob. Lett. 30(4), 313-319 (1996b)

42. Wilfling, B.: A sufficient condition for Lorenz ordering. Sankhyā: The Indian Journal of Statistics. Series B 62-69 (1996c)

43. Wilfling, B., Krämer, W.: The Lorenz-ordering of Singh-Maddala income distributions. Econ. Lett. 43(1), 53-57 (1993)

44. van Zwet, W.R.: Convex transformations of random variables. Mathematisch Centrum (1964)

Publisher's Note Springer Nature remains neutral with regard to jurisdictional claims in published maps and institutional affiliations. 